\title{
Comparação da percepção de qualidade de vida entre adolescentes praticantes e não praticantes de esporte no contraturno escolar
}

\section{Comparison of the perception of quality of life among adolescents who do and do not participate in sports in school counterpart}

\author{
Ana Beatriz Pacífico' (D), Anelize Gabriele Peressute ${ }^{1}$ (D), Thiago Silva Piola ${ }^{1}$ (D), \\ Edina Maria de Camargo ${ }^{1}$ (D), Wagner de Campos $^{1}$ (D) \\ ${ }^{1}$ Centro de Estudos em Atividade Física e Saúde (CEAFS), Universidade Federal do Paraná (UFPR) - Curitiba (PR), Brasil.
}

\begin{abstract}
Como citar: Pacífico AB, Peressute AG, Piola TS, Camargo EM, Campos W. Comparação da percepção de qualidade de vida entre adolescentes praticantes e não praticantes de esporte no contraturno escolar. Cad Saúde Colet, 2020;28(4):548-555. https://doi.org/10.1590/1414-462X202028040481
\end{abstract}

\section{Resumo}

Introdução: O esporte pode contribuir com a percepção de qualidade de vida dos adolescentes nessa fase de desenvolvimento. Objetivo: Comparar os domínios da percepção de qualidade de vida de adolescentes, dos sexos masculino e feminino, praticantes de esporte no contraturno escolar (PE), praticantes de outra modalidade de exercício físico (PMEF) e não praticantes de exercício físico (NPEF). Método: O estudo é transversal com delineamento ex post facto, com a população composta por adolescentes e com uma amostra de 374 sujeitos, dividida em três grupos. Para avaliar a percepção de qualidade de vida dos adolescentes, foi utilizado o questionário KIDSCREEN-52, e para a análise comparativa, o teste da Análise de variância (ANOVA) de um fator com post hoc de Scheffe. Resultados: As comparações das dimensões de qualidade de vida (QV) mostraram que a prática esportiva é um importante fator para melhores percepções de QV e, consequentemente, uma melhor percepção de bem-estar, isto principalmente para o sexo masculino ( $F=5,38 ; 3,86 ; 4,79 ; 6,22 ; 7,93$, para sentimentos; estado emocional; autopercepção; amigos; apoio social, respectivamente, $\mathrm{p}<0,05)$. Conclusão: A prática esportiva, em especial na adolescência, merece destaque, já que os praticantes de esporte no contraturno apresentaram melhores percepções de QV nas diferentes dimensões analisadas.

Palavras-chave: esporte; percepção de qualidade de vida; adolescente.

\section{Abstract}

Background: Sports can enhance adolescents' quality of life perception in their important development phase. Objective: compare the domains of quality of life perception of adolescents, male and female, who participate in sports (SP), or another type of physical exercise (PTPE) and non-physical exercise practitioners (NPEP) in the school counterpart. Method: The study is cross-sectional with ex post facto design, and its population is composed of adolescents, with a total sample of 374 subjects, divided into three groups (SP, PTPE, NPEP). To evaluate the perception of quality of life of the adolescents, the KIDSCREEN-52 questionnaire was used, and for the comparative analysis of the data, the Analysis of variance (ANOVA) test of a factor with post hoc of Schefe. Results: The comparison of quality of life (QoL) dimensions showed that sports practice is an important factor for better perceptions of QoL, consequently a better perception of well-being, especially for males $(F=5.38,3.86,4.79,6.22,7.93$, for feelings, emotional state, self perception, friends, social support respectively and with $\mathrm{p}<0.05)$. Conclusion: The sport practice, especially in adolescence, deserves to be highlighted, since those who participate in sports in the counterpart presented significant and better perceptions of QoL in the different dimensions analyzed.

Keywords: sport; perception of quality of life; adolescent.

Este é um artigo publicado em acesso aberto (Open Access) sob a licença Creative Commons Attribution, que permite uso, distribuição e reprodução em qualquer meio, sem restrições desde que o trabalho original seja corretamente citado. 


\section{INTRODUÇÃO}

A prática de atividades físicas e esporte, e a participação de aulas na educação física escolar são elementos importantes para influenciar uma vida saudável na adolescência'. Para uma vida saudável, também é importante que o indivíduo apresente uma boa percepção de qualidade de vida (QV), entusiasmando o sujeito a construir determinados parâmetros da sua própria vida social e emocional também. Além disso, a QV está voltada a menores números de morbimortalidades ${ }^{2,3}$.

O comportamento semanal de altos níveis de atividade física se relaciona à percepção da QV, embora uma minoria seja considerada fisicamente ativa suficientemente. A baixa adesão à prática de atividade física está relacionada a um pior desempenho escolar, baixa autoestima, exclusão social e baixa percepção de qualidade de vida. E, quanto ao sexo, as meninas apresentam pior percepção de qualidade de vida ${ }^{4}$.

Na adolescência, a percepção de QV pode ser afetada mais facilmente ${ }^{5}$, pois esta fase é considerada como vulnerável, devido a intensos momentos de transição e desenvolvimento do adolescente. Assim, é de extrema relevância a monitoração da percepção de QV dos adolescentes, sendo que esta percepção pode repercutir na idade adulta ${ }^{6}$.

A escola pode ser um ambiente mediador e promotor de ações voltadas para uma melhor percepção de $\mathrm{QV}$, uma vez que é um dos ambientes onde o adolescente passa grande parte do seu dia $^{7}$. Nesse contexto, o ambiente pode proporcionar atividades esportivas, socialização nas aulas de educação física e incentivo à prática de exercícios físicos em horários extracurriculares ${ }^{8}$. Dentre a oferta de atividades extracurriculares, está o esporte, que, segundo Gáspari \& Schwartz ${ }^{2}$, é um elemento facilitador para melhoria da percepção de qualidade de vida, além de contemplar a automotivação e oportunizar situações capazes de gerar mudanças atitudinais. Os adolescentes que passam mais tempo praticando esportes em equipe ou individual, que dormem no tempo adequado e que têm elevados níveis de atividade física estão positivamente associados a uma melhor percepção de QV quando comparados com adolescentes com altos níveis de sedentarismo 9,10.

Embora seja crescente o número de publicações que contemplem a qualidade de vida, poucos estudos são relacionados à saúde do adolescente no Brasil, sendo que a maioria destes estudos avalia a percepção de qualidade de vida em crianças e adolescentes como uma medida clínica. Também há estudos que analisam a prática de atividades físicas dos adolescentes, entre as quais a prática do esporte, investigando aspectos psicossociais, qualidade de vida em indivíduos considerados saudáveis e qualidade de vida em indivíduos com deficiência ou lesionados ${ }^{11-14}$.

Assim, são necessários estudos que abordem a percepção de qualidade de vida, valorizando a perspectiva de adolescentes na sua fase de desenvolvimento. Diante disso, o objetivo do presente estudo foi comparar os domínios da percepção de qualidade de vida de adolescentes, dos sexos masculino e feminino, praticantes de esporte, praticantes de outra modalidade de exercício físico e não praticantes de exercício físico no contraturno escolar.

\section{MÉTODO}

Trata-se de um estudo transversal com delineamento ex post facto. $O$ estudo seguiu as normas que do Conselho Nacional de Saúde (resolução n 466/2012), aprovado pelo Comitê de Ética em Pesquisa da UFPR, sob registro CAAE: 66930117.8 .0000 .0102 , sendo que a coleta de dados foi realizada em 2017.

A população do estudo foi formada por adolescentes do Ensino Médio, estudantes das escolas particulares de Curitiba que ofertam atividades no contraturno com foco esportivo, sendo uma amostra intencional. Foi realizado o cálculo amostral a posteriori para três grupos, uma vez que o estudo consiste na comparação de variáveis de praticantes de esporte, praticantes de outra modalidade de exercício físico e não praticantes de exercício físico, considerando um effect size de $\eta^{2}=0,3$, o nível de significância de 0,05 e o total de 374 sujeitos, obtendo um poder amostral de 0,99. 
A amostra foi dividida em três grupos: i) PE - praticantes de esportes (participantes dos treinos esportivos oferecidos pela escola); ii) PMEF - praticantes de outra modalidade de exercício físico (praticantes de outra atividade física sistematizada, fora da escola ou dentro da escola, mas que não fosse esporte), e iii) NPEF - não praticantes de exercício físico (não praticantes de atividade física sistematizada). Para serem classificados como PE ou PMEF, o adolescente teria que praticar o exercício pelo menos duas vezes por semana, ou então ser considerado como NPEF, além de praticar ou só o esporte no contraturno ou só o exercício físico, para que a classificação fosse analisada sem sobreposição.

A pesquisa teve como critérios de inclusão: i) alunos matriculados nas escolas participantes; ii) ter 15 anos completos e menos de 18 anos; iii) aceitar participar do estudo, através da assinatura do Termo de Consentimento Livre e Esclarecido (TCLE) pelos pais ou responsáveis, e iv) assinar o Termo de Assentimento (TA) pelos adolescentes. Adotaram-se como critérios de exclusão: i) os adolescentes com deficiência física ou mental; ii) não concluir todo o questionário; iii) ser praticante de esporte no contraturno e outro exercício físico, e iv) ser praticante de esporte no contraturno que não fosse oferecido pela escola.

Foi realizado um estudo piloto e depois a pesquisa foi realizada nas escolas que concordaram em participar. O estudo foi feito durante as aulas de Educação Física, sendo que os questionários foram preenchidos com o acompanhamento da equipe de coleta e do professor da escola.

A variável independente - praticar ou não esporte no contraturno - foi obtida por questionário de anamnese, no qual o adolescente era questionado sobre praticar esporte no contraturno ou praticar outra modalidade de exercício físico oferecido pela escola ou não. A variável dependente do estudo foi a percepção de qualidade de vida separada por domínios: Saúde e atividade física; Sentimentos; Estado emocional; Autopercepção; Tempo livre; Ambiente familiar; Aspecto financeiro; Amigos e apoio social; Ambiente escolar, e Provocação do Bullying. Para caracterização da amostra, ainda foram coletadas informações, como sexo e idade.

Para avaliar a percepção de qualidade de vida dos adolescentes, foi utilizado o questionário KIDSCREEN-52, validado no Brasil ${ }^{6}$. Neste questionário, o a de Cronbach apresenta coeficientes entre 0,725 e 0,894 . Os valores de consistência interna na versão criança/adolescente variaram entre 0,725 na dimensão "Autopercepção" e 0,894 na dimensão "Aspecto Financeiro", com valor global médio de $0,817^{6}$. As questões são apresentadas em escala likert, abrangendo dez dimensões da qualidade de vida.

Para análise dos dados, foi estabelecido um nível de significância estabelecido em $p<0,05$. Foi utilizada a distribuição de frequência absoluta e relativa, e o teste de qui-quadrado foi empregado para a caracterização da amostra. Ainda, na análise comparativa, foi realizada uma comparação através do teste da análise de variância (ANOVA) de um fator com post hoc de Schefe. A análise foi realizada pelo programa estatístico Statistical Package for the Social Sciences ${ }^{\circledR}$ versão 20.0 (SPSS ${ }^{\circledR}$ Inc., Chicago, IL, EUA).

\section{RESULTADOS}

O presente estudo teve como amostra 374 adolescentes, 198 meninos e 176 meninas, sendo as faixas etárias $16,35 \pm 0,65$ anos e 16,19 $\pm 0,67$ anos, respectivamente. A maior parte dos meninos (54,5\%) foram classificados como praticantes de esporte (PE) e 17,6\% das meninas se classificaram como PE. Como praticante de outra modalidade de exercício físico (PMEF), foram classificados $18,2 \%$ dos meninos e $26,7 \%$ das meninas. A maior parte das meninas da amostra foram classificadas como não praticantes de exercício físico (NPEF) com 55,7\%, e os meninos com 27,3\% (Tabela 1). Como pode ser observado na Tabela 1, não houve diferença significativa entre os sexos e entre os grupos (PE, PMEF e NPEF) na proporção de idade.

Na percepção de QV (a partir de um escore geral dos domínios e dividido em tercis), a maioria dos adolescentes foi classificada como baixa percepção de QV (33,7\%). O grupo NPEF apresentou diferença significativa entre os sexos $(p=0,022)$, em que mais meninas apresentaram baixa percepção de $\mathrm{QV}$ do que os meninos, observando-se que o mesmo não aconteceu nos demais grupos, quando investigada a diferença entre os sexos. No grupo $\mathrm{PE}$, a maior frequência 


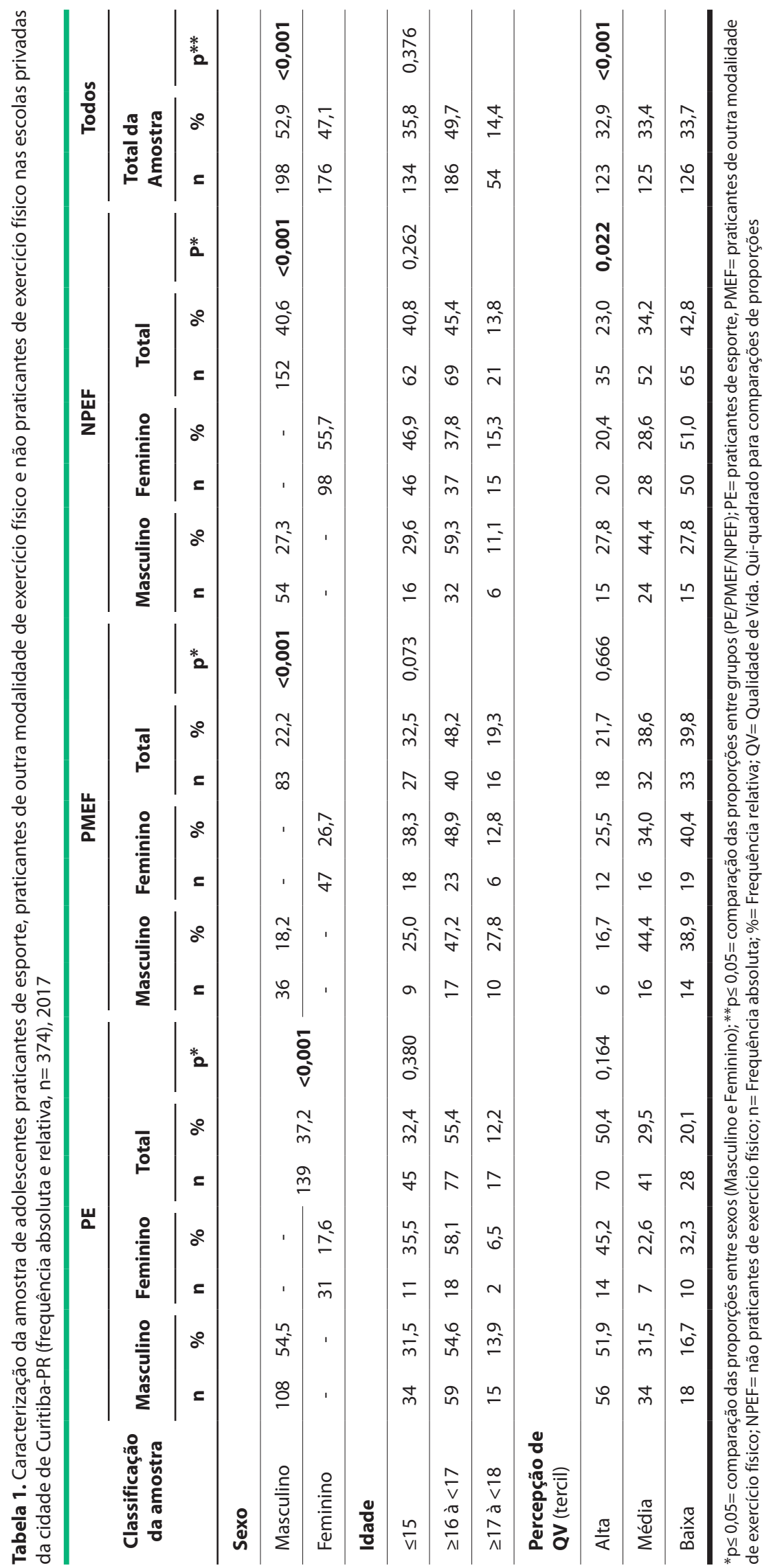


Tabela 2. Comparação entre praticantes de esporte, praticantes de outra modalidade de exercício físico e não praticantes de exercício físico em relação às dimensões da percepção de qualidade de vida, de acordo com o kidscreen, para o sexo masculino $(n=198)$, Curitba - PR, 2017

\begin{tabular}{|c|c|c|c|c|}
\hline & PE & PMEF & NPEF & \\
\hline & $(n=108)$ & $(n=36)$ & $(n=54)$ & $\mathbf{F}$ \\
\hline & Média \pm DP & Média \pm DP & Média \pm DP & \\
\hline Saúde e AF & $74,11 \pm 17,38$ & $71,78 \pm 13,28$ & $61,48 \pm 12,54$ & 0,78 \\
\hline Sentimentos & $79,26 \pm 13,98$ & $70,46 \pm 17,17^{\beta}$ & $75,06 \pm 13,36^{\beta}$ & $5,38 * *$ \\
\hline Estado emocional & $76,11 \pm 14,03$ & $68,89 \pm 13,64^{\beta}$ & $74,76 \pm 12,42$ & $3,86^{*}$ \\
\hline Autopercepção & $71,56 \pm 12,71$ & $64,44 \pm 13,94^{\beta}$ & $72,07 \pm 12,22^{¥}$ & $4,79^{* *}$ \\
\hline Tempo livre & $77,18 \pm 13,21$ & $71,44 \pm 12,10^{\beta}$ & $69,62 \pm 15,78^{\beta}$ & $6,22 * *$ \\
\hline Ambiente familiar & $79,23 \pm 14,60$ & $74,17 \pm 14,46$ & $77,16 \pm 13,22$ & 1,77 \\
\hline Aspecto financeiro & $76,48 \pm 17,09$ & $75,00 \pm 21,34$ & $77,77 \pm 15,86$ & 0,27 \\
\hline Amigos e apoio social & $83,06 \pm 12,45$ & $77,31 \pm 31,48$ & $74,44 \pm 15,29^{\beta}$ & $7,93 * *$ \\
\hline Ambiente escolar & $69,26 \pm 16,00$ & $66,85 \pm 12,99$ & $69,20 \pm 11,65$ & 0,40 \\
\hline Provocação de Bullying & $88,82 \pm 12,12$ & $87,03 \pm 13,52$ & $90,24 \pm 10,49$ & 0,78 \\
\hline
\end{tabular}

${ }^{*} p \leq 0,05 ;{ }^{* *} p \leq 0,01 ; F=$ ANOVA a um fator, Post Hoc Scheffe; $\beta$ Diferente de quem pratica esporte; $¥$ Diferente de quem pratica outra modalidade de exercício físico; $\mathrm{PE}=$ praticantes de esporte; $\mathrm{PMEF}=$ praticantes de outra modalidade de exercício físico; $\mathrm{NPEF}=$ não praticantes de exercício físico; $\mathrm{DP}=$ Desvio Padrão; $\mathrm{AF}=$ Atividade Física; $\mathrm{n}=$ número de indivíduos.

dos adolescentes foi no tercil alto de percepção de QV (51,9\% e 45,2\%, meninos e meninas, respectivamente), o que não aconteceu nos demais grupos ( $p<0,001$ ) (Tabela 1).

A Tabela 2 apresenta a média e o desvio padrão da comparação entre praticantes de esporte, praticantes de outra modalidade de esporte e não praticantes de exercício físico, em relação às dimensões da percepção de QV para o sexo masculino. Os escores da maioria das dimensões dos PE foram maiores do que as médias dos PMEF e NPEF. A dimensão dos sentimentos apresentou escore menor $(p \leq 0,01)$ para os NPEF e para os PMEF, sendo ambos comparados aos $\mathrm{PE}$. $\mathrm{O}$ estado emocional foi significativamente maior $(p \leq 0,05)$ para os $\mathrm{PE}$, quando comparados aos PMEF. A dimensão autopercepção apresentou diferença $(p \leq 0,01)$ entre os grupos, apresentando-se maior para os $\mathrm{PE}$ comparados aos $\mathrm{PMEF}$, e maior para os NPEF comparados aos PMEF. A dimensão do tempo livre obteve um escore maior $(p \leq 0,01)$ para os PE comparados tanto com os PMEF quanto com os NPEF. A outra dimensão que apresentou valores significativos $(p \leq 0,01)$ foi a dimensão amigos e apoio social, sendo maior para os PE e menor para os NPEF.

A Tabela 3 apresenta a comparação entre praticantes de esporte, praticantes de outra modalidade de exercício físico e não praticantes de exercício físico, em relação às dimensões da percepção de QV para o sexo feminino. Apenas duas dimensões apresentaram diferenças estatísticas entre os escores, sendo as médias maiores para as praticantes de esporte. Os escores dos sentimentos foram diferentes significativamente $(p \leq 0,05)$ entre as PE e as NPEF. $E$ a outra dimensão que apresentou diferença $(p \leq 0,01)$ foi a do estado emocional, em que as PE obtiveram maiores escores comparadas às PMEF e às NPEF.

\section{DISCUSSÃO}

Os resultados da comparação das dimensões de QV, de acordo com o questionário de percepção de QV, o KIDSCREEN, mostraram que a prática esportiva é um importante fator para melhores percepções de QV e, consequentemente, uma melhor percepção de bem-estar. Isto ocorre principalmente para o sexo masculino, segundo os resultados do presente estudo. Para os meninos, de cinco dimensões que apresentaram significância na 
Tabela 3. Comparação entre praticantes de esporte, praticantes de outra modalidade de exercício físico e não praticantes de exercício físico em relação às dimensões da percepção de qualidade de vida, de acordo com o kidscreen, para o sexo feminino ( $n=176)$, Curitba - PR, 2017

\begin{tabular}{|c|c|c|c|c|}
\hline & PE & PMEF & NPEF & \\
\hline & $(n=31)$ & $(n=47)$ & $(n=98)$ & $\mathbf{F}$ \\
\hline & Média \pm DP & Média \pm DP & Média \pm DP & \\
\hline Saúde e AF & $71,10 \pm 16,09$ & $65,79 \pm 11,79$ & $54,49 \pm 13,26$ & 0,55 \\
\hline Sentimentos & $77,74 \pm 15,16$ & $70,85 \pm 16,77^{\beta}$ & $68,70 \pm 16,46^{\beta}$ & $3,60^{*}$ \\
\hline Estado emocional & $70,41 \pm 16,69$ & $67,17 \pm 15,45^{\beta}$ & $63,44 \pm 15,54^{\beta}$ & $2,61 *$ \\
\hline Autopercepção & $66,06 \pm 15,03$ & $63,23 \pm 15,59$ & $61,26 \pm 15,16$ & 1,22 \\
\hline Tempo livre & $67,35 \pm 14,57$ & $69,11 \pm 13,18$ & $68,85 \pm 12,70$ & 0,19 \\
\hline Ambiente familiar & $74,40 \pm 19,69$ & $73,54 \pm 17,82$ & $73,26 \pm 17,74$ & 0,05 \\
\hline Aspecto financeiro & $76,55 \pm 16,67$ & $75,74 \pm 20,21$ & $74,15 \pm 19,81$ & 0,23 \\
\hline Amigos e apoio social & $80,21 \pm 13,25$ & $76,31 \pm 14,86$ & $78,29 \pm 12,76$ & 0,81 \\
\hline Ambiente escolar & $71,83 \pm 15,20$ & $68,58 \pm 13,74$ & $67,14 \pm 14,93$ & 1,21 \\
\hline Provocação de Bullying & $92,68 \pm 9,94$ & $91,49 \pm 10,65$ & $90,20 \pm 13,37$ & 0,55 \\
\hline
\end{tabular}

${ }^{*} \mathrm{p} \leq 0,05 ; \mathrm{F}=\mathrm{ANOVA}$ a um fator, Post Hoc Scheffe; $\beta$ Diferente de quem pratica esporte; $\mathrm{n}=$ número de indivíduos; $\mathrm{PE}=$ praticantes de esporte, $\mathrm{PMEF}=$ praticantes de outra modalidade de exercício físico; NPEF= não praticantes de exercício físico; $\mathrm{DP}=$ Desvio Padrão; $\mathrm{AF}=$ Atividade Física

comparação (Sentimentos; Estado Nutricional, Autopercepção; Tempo livre; Amigos e apoio social), três são melhores para os praticantes de esporte (Sentimentos; Tempo livre; Amigos e apoio social) quando comparadas com os outros dois grupos, e uma (autopercepção) é melhor para esportistas ao ser comparada com praticantes de outro exercício físico. As duas dimensões que foram significativas para as meninas (Sentimentos; Estado emocional) são melhores para aquelas que praticam esporte, comparadas com praticantes de outra modalidade de exercício físico e de não praticantes de exercício físico.

Estes resultados vão ao encontro com os achados de Cevada ${ }^{15}$, que, através de um estudo que relaciona o esporte com a resiliência, a qualidade de vida e a ansiedade de adolescentes, comprovam que há diferença na percepção de aspectos emocionais da QV, na resiliência e nos aspectos emocionais de indivíduos que praticam esporte, com valores maiores quando comparados com os indivíduos que não são praticantes de esportivo.

Em um estudo de associação, realizado na Espanha, com adolescentes, em que foi utilizado o mesmo questionário desta pesquisa para avaliar a percepção de QV, o KIDSCREEN, mostrou-se que a AF está positivamente associada com a percepção de $\mathrm{QV}$ relacionada à saúde, em que a AF representou $11,3 \%$ de maiores níveis de percepção de QV. Porém, este estudo não especificou quais eram as atividades físicas realizadas pelos adolescentes ${ }^{16}$. É importante destacar que, quando se trata de adolescentes atletas profissionais e não aqueles praticantes de esporte oferecidos pela escola, ou seja, como lazer, a percepção de QV pode ser prejudicada, como, por exemplo, no estudo de Britton e autores ${ }^{13}$, que avalia atletas profissionais, em que se verificou que esta prática está associada negativamente com a estabilidade emocional e a satisfação com a vida.

Assim como nos achados de Ferreira et al. ${ }^{17}$, que reportam que praticantes de esporte apresentam maiores dores e, relacionada a isso, uma menor percepção de QV, o que contradiz os achados do presente estudo. Esta diferença entre os achados pode ser justificada pelo fato de o estudo de Ferreira e colaboradores ter sido realizado com atletas em treinamentos esportivos, cujos fins são geralmente chegar ao limite do indivíduo e os resultados são cobrados de uma forma mais rígida do que nos treinos de contraturnos oferecidos pela escola (como no 
presente estudo). Estes treinos na escola parecem deixar a prática mais prazerosa e menos lesiva, podendo resultar em uma melhor percepção de QV quando comparados com o treinamento esportivo focado na formação de atletas profissionais, em que há grandes índices de lesão e dor.

É importante destacar que poucas dimensões apresentaram diferenças estatísticas, principalmente para o sexo feminino, o que pode ter relação com o que o estudo de Guedes e Silvério ${ }^{18}$, com 1.517 sujeitos, apresenta, ao mostrar que os principais motivos que levam os adolescentes a praticar esporte são a competência técnica e a aptidão física, e os motivos menos importantes para os adolescentes são diversão e reconhecimento social.

Diante dos resultados, a prática esportiva, principalmente na adolescência, merece destaque, já que os praticantes de esporte no contraturno apresentaram significativas e melhores percepções de QV nas diferentes dimensões, percepções estas que foram diferentes entre os grupos. Então, sugere-se que haja sempre o incentivo ao esporte, com atenção em como são proporcionados os treinos, se eles estão sendo suficientes para trazer benefícios aos adolescentes ou se ainda é possível melhorar a forma da prática esportiva oferecida pelas escolas. Esta atenção precisa ser redobrada para as meninas, pois poucas diferenças foram observadas entre elas, sendo necessário incentivar ainda mais as adolescentes, oferecer mais opções a elas e adequar os treinamentos ao sexo feminino, também.

Ainda, ao se tratar do esporte, é relevante mencionar que a prática está suscetível a lesões e dores crônicas $^{19}$, o que pode causar afastamento do indivíduo na prática esportiva. Diante disso, recomenta-se que, na adolescência, além do treino de esporte, haja um acompanhamento e um trabalho em paralelo supervisionando e instruindo os adolescentes a realizar exercícios que possam prevenir lesões e recuperar-se de alguma possível dor ${ }^{20}$. Pode-se, assim, evitar que o adolescente se afaste do esporte.

O estudo apresentou algumas limitações, dentre as quais a pesquisa ser realizada somente com escolas particulares da cidade de Curitiba, a amostra ser intencional e não representativa de grande população, os resultados das variáveis NAF e a percepção de QV terem sido obtidos através de questionários, o que, apesar de os instrumentos apresentarem boa reprodutibilidade, validade concorrente e validade interna, pode superestimar ou subestimar os valores. Assim, para minimizar esses vieses, os pesquisadores foram treinados para auxiliar os alunos a responder os questionários da melhor forma.

Sugerem-se, então, novas pesquisas que analisem o volume, a intensidade e a frequência da prática de esporte e a prática de outras $\mathrm{AF}$, além da utilização de outros tipos de instrumentos, como o acelerômetro, para avaliar o nível de AF. São necessárias análises dos treinos esportivos que são disponibilizadas no contraturno escolar aos adolescentes, investigando quais e como estes são oferecidos a eles.

Os praticantes de esporte no contraturno possuem melhor percepção de qualidade de vida na maioria dos domínios, a qual apresentou diferença significativa entre os grupos, quando comparados aos praticantes de outra modalidade de exercício físico e aos não praticantes de exercício físico, nas dimensões da QV.

Os achados do estudo mostram a relevância da prática esportiva na adolescência e, para que isso seja possível e eficaz, o contraturno esportivo nas escolas precisa ser oferecido e ser de qualidade, tanto para meninos quanto para meninas. Neste estudo, a maior parte dos adolescentes do sexo masculino do estudo foi classificada como praticantes de esporte, enquanto a maioria das meninas foi classificada como não praticantes de exercício físico, o que mostra a importância de uma atenção maior para as meninas. Por fim, é possível afirmar que ainda é preciso que os adolescentes apresentem melhores resultados em algumas dimensões da percepção de QV.

\section{REFERÊNCIAS}

1. World Health Organization. Global recommendations on physical activity for health. Geneva:WHO; 2010. p. 1-60.

2. Gáspari JC, Schwartz GM. Adolescência, esporte e qualidade de vida. Motriz. 2001;7:107-13. 
3. Gaspar T, Matos MG. Qualidade de vida em crianças e adolescentes versão portugues dos Instrumentos Kidscreen-52. United Kingdom: Health Behaviour in School-aged Children; 2008.

4. Lima-serrano M, Martínez-montilla JM, Guerra-martín MD, Vargas-Martínez AM, Lima-Rodríguez JS. Factores relacionados con la calidad de vida en la adolescencia. Gac Sanit. 2016;32(1):68-71. http://dx.doi. org/10.1016/j.gaceta.2016.06.016. PMid:27567181.

5. Fonseca FF, Sena RKR, Santos RLA, Dias OV, Costa SM. As vulnerabilidades na infância e adolescência e as políticas públicas brasileiras de intervenção. Rev Paul Pediatr. 2013 jun;31(2):258-64. http://dx.doi. org/10.1590/S0103-05822013000200019. PMid:23828065.

6. Guedes DP, Guedes JERP. Tradução, adaptação transcultural e propriedades psicométricas do KIDSCREEN-52 para a população brasileira. Rev Paul Pediatr. 2011;29(3):364-71. http://dx.doi.org/10.1590/ S0103-05822011000300010.

7. Agathão $\mathrm{BT}$, Reichenheim ME, Moraes CL. Qualidade de vida relacionada à saúde de adolescentes escolares. Cien Saude Colet. 2018;23(2):659-68. http://dx.doi.org/10.1590/1413-81232018232.27572016. PMid:29412423.

8. Gordia AP, Quadros TMB, Silva RCR, Campos WD. Domínio social da qualidade de vida de adolescentes e sua associação com variáveis comportamentais, biológicas e sociodemográficas. Rev Educ Fis UEM. 2015;26(3):451-63. http://dx.doi.org/10.4025/reveducfis.v26i3.23066.

9. Casey M, Harvey J, Telford A, Eime R, Mooney A, Payne W. Patterns of time use among regional and rural adolescent girls: associations with correlates of physical activity and health-related quality of life. J Sci Med Sport. 2016 nov;19(11):931-5. http://dx.doi.org/10.1016/j.jsams.2016.02.004. PMid:26996947.

10. Nascimento MMR, Melo TR, Pinto RMC, Morales NM, Mendonça TM, Paro HB, et al. Parents' perception of health-related quality of life in children and adolescents with excess weight. J Pediatr (Rio J). 2016;92(1):6572. http://dx.doi.org/10.1016/j.jped.2015.04.006. PMid:26397741.

11. Balassiano DH, Araújo CGS. Maximal heart rate: influence of sport practice during childhood and adolescence. Arq Bras Cardiol. 2013;100(4):333-8. http://dx.doi.org/10.5935/abc.20130055. PMid:23545996.

12. Minatto G, Silva DAS, Pelegrini A, Fidelix YL, Silva AF, Petroski EL. Aptidão cardiorrespiratória, indicadores sociodemográficos e estado nutricional em adolescentes. Rev Bras Med Esporte. 2015;21(1):12-6. http:// dx.doi.org/10.1590/1517-86922015210101385.

13. Britton D, Kavanagh E, Polman R. The Perceived Stress Reactivity Scale for adolescent athletes. Pers Individ Dif. 2017;116:301-8. http://dx.doi.org/10.1016/j.paid.2017.05.008.

14. Caronni A, Sciumè L, Donzelli S, Zaina F, Negrini S. ISYQOL: a Rasch-consistent questionnaire for measuring health-related quality of life in adolescents with spinal deformities. Spine J. 2017 set;17(9):1364-72. http:// dx.doi.org/10.1016/j.spinee.2017.05.022. PMid:28529002.

15. Cevada T, Cerqueira LS, Moraes HS, Santos TM, Pompeu FAMS, Deslandes AC. Relação entre esporte, resiliência, qualidade de vida e ansiedade. Rev Psiquiatr Clin (Santiago). 2012;39(3):85-9. http://dx.doi. org/10.1590/S0101-60832012000300003.

16. Muros JJ, Salvador Pérez F, Zurita Ortega F, Gámez Sánchez VM, Knox E. The association between healthy lifestyle behaviors and health-related quality of life among adolescents. J Pediatr (Rio J). 2017;93(4):40612. http://dx.doi.org/10.1016/j.jped.2016.10.005. PMid:28130968.

17. Ferreira AS, de Oliveira Silva D, Priore LBD, Garcia CLG, Ducatti MHM, Botta AFB, et al. Differences in pain and function between adolescent athletes and physically active non-athletes with patellofemoral pain. Phys Ther Sport. 2018;33:70-5. http://dx.doi.org/10.1016/j.ptsp.2018.07.005. PMid:30025378.

18. Guedes DP, Silvério JE No. Motivos para a prática de esportes em atletas jovens e fatores associados. Rev Educ Fis UEM. 2013;24(1):21-31. http://dx.doi.org/10.4025/reveducfis.v24i1.14695.

19. Esteve E, Rathleff MS, Bagur-Calafat C, Urrútia G, Thorborg K. Prevention of groin injuries in sports: a systematic review with meta-analysis of randomised controlled trials. Br J Sports Med. 2015;49(12):78591. http://dx.doi.org/10.1136/bjsports-2014-094162. PMid:25730819.

20. Rathleff MS, Roos EM, Olesen JL, Rasmussen S. Exercise during school hours when added to patient education improves outcome for 2 years in adolescent patellofemoral pain: a cluster randomised trial. Br J Sports Med. 2015;49(6):406-12. http://dx.doi.org/10.1136/bjsports-2014-093929. PMid:25388552. 